\title{
The stability of the orbits of Earth-mass planets in the habitable zone of 47 Ursae Majoris
}

\author{
B. W. Jones and P. N. Sleep \\ Astronomy Group, The Open University, Milton Keynes, MK7 6AA, UK \\ Received 11 April 2002 / Accepted 1 August 2002

\begin{abstract}
We have investigated whether Earth-mass planets could survive in the habitable zone (HZ) of the 47 Ursae Majoris system. Mixed-variable symplectic numerical integration has been used to investigate the orbits of putative Earth-mass planets. Whereas the 47 UMa system as previously known, with just one giant planet, could have Earth-mass planets that remain confined to the $\mathrm{HZ}$ for a fairly wide range of initial obital parameters, the second (outer) giant now known to be present has reduced the range significantly. There are however confined orbits particularly if either the present eccentricity of the outer giant is close to zero, or the giant masses are close to the minimum values. We have also shown that the eccentricity of the outer giant's orbit is unlikely to exceed about 0.15 at minimum giant mass and 0.13 at 1.5 times this minimum.
\end{abstract}

Key words. stars: planetary systems - planets and satellites: general

\section{Introduction}

The habitable zone (HZ) is the range of distances from a star within which water at the surface of a terrestrial planet would be in the liquid phase. The question we have addressed is: "could planets with masses of the order that of the Earth survive in the HZ of the 47 Ursae Majoris system?". If they could survive for the order of the present age of the star then such planets might be present, and if they are terrestrial in composition they would be potential habitats for exobiospheres.

About 90 exoplanetary systems (exosystems) are now known (Schneider 2002). One that approximates the Solar System is the 47 UMa system, in that the giants are comparatively far from the star and are in low eccentricity orbits. As is the case for most exoplanetary systems, $47 \mathrm{UMa}$ is a solar-type star. The system properties are summarised in Table 1, with Solar System properties for comparison (Fischer et al. 2002). In this table $m$ is the mass of the giant (in Jupiter masses $m_{\mathrm{J}}$ ), $a$ is the semimajor axis of the orbit, and $e$ is its orbital eccentricity. The eccentricity of the outer giant's orbit is not well known, so we have investigated the stability of the system to place an upper limit on this value. The giant masses in Table 1 are the minimum values, as revealed through the Doppler technique by which the planets were discovered. This technique gives $m \sin \left(i_{0}\right)$, where $m$ is the mass of the planet and $i_{0}$ is the inclination of its orbital plane with respect to the plane of the sky. Gonzalez (1998), from the absorption lines of $47 \mathrm{UMa}$, obtained an estimate of $44^{\circ}$ with uncertainties of $+24^{\circ} /-22^{\circ}$

Send offprint requests to: B. W. Jones,

e-mail: B.W. Jones@open.ac.uk for the axial inclination of the star, and so if the planets orbit in the star's equatorial plane then $i_{0} \approx 44^{\circ}$ and the actual planetary masses would be about 1.4 times the $m \sin \left(i_{0}\right)$ values in Table 1 . Pourbaix (2001) has used Hipparcos data to obtain a similar value for the multiplier, also with low confidence. Perryman et al. (1996) have used Hipparcos data to set a lower limit of $i_{0}=20^{\circ}$ for the inner giant, in which case the multiplier would be less than 3 . For random orientation on the sky the multiplier, on average, is 1.3.

It is believed (Lissauer 1987; Pollack et al. 1996) that all the known exoplanets are rich in hydrogen and helium, and therefore resemble the giant planet Jupiter rather than a class unknown in our Solar System, namely, the supermassive terrestrial planet (the Earth has a mass of only $3.15 \times$ $10^{-3} m_{\mathrm{J}}$ ). This belief is supported by the radius of the $0.69 \mathrm{~m}_{\mathrm{J}}$ planet HD 209458b, which has been determined from its transits (Charbonneau et al. 2000). The value is about $1.35 R_{\mathrm{J}}$, where $R_{\mathrm{J}}$ is the radius of Jupiter. Given that the known exoplanets are Jupiter-like, they probably formed not where they are now, but further from the star, and then migrated inwards (Boss 1995).

Various migration schemes have been proposed. It is expected that migration would have left the zone traversed by the giant, and much of the space interior to this zone, devoid of Earth-mass bodies. However, if migration is caused either by planetesimal scattering by the giant (for example, Murray et al. 1998), or by the interaction of the giant with the nebular disc (for example Lin et al. 2000; Ward \& Hahn 2000; Masset \& Snellgrove 2001), it is possible that there was enough material left over after giant migration to create Earth-mass 
Table 1. The 47 Ursae Majoris system with one and two giants, and the Solar System.

\begin{tabular}{|c|c|c|c|c|c|c|c|}
\hline \multicolumn{3}{|c|}{ star } & \multicolumn{5}{|c|}{ planets } \\
\hline name & type & $\operatorname{mass} / M_{\odot}$ & name & $m \sin \left(i_{\circ}\right)$ & $a / \mathrm{AU}$ & $e$ & $\begin{array}{l}i \\
/ \operatorname{deg}\end{array}$ \\
\hline $47 \mathrm{UMa}$ & G1V & 1.03 & G1 & 2.54 & 2.09 & $0.061 \pm 0.014$ & 0 (by defn) \\
\hline & & & $\mathrm{G} 2$ & 0.76 & 3.73 & $0-0.2$ & low? \\
\hline $47 \mathrm{UMa}$ & G1V & 1.03 & $\mathrm{G}$ & 2.41 & 2.10 & $0.096 \pm 0.03$ & 0 (by defn) \\
\hline Sun & G2V & 1.00 & Jupiter & 1 & 5.203 & 0.0489 & 1.304 \\
\hline & & & Saturn & 0.299 & 9.586 & 0.0576 & 2.486 \\
\hline
\end{tabular}

planets. Laughlin et al. (2002) have shown that with the giants in $47 \mathrm{UMa}$ in their present orbits it is possible to build up Earth-mass planets within about $0.7 \mathrm{AU}$ of the star, and they do not rule out formation at greater distances, extending into the present-day habitable zone.

Earth-mass planets have not yet been detected, and so one can only theorise about their existence. We have placed an Earth-mass planet at various distances from $47 \mathrm{UMa}$, but always within or near the habitable zone. Whether life could have emerged on an Earth-mass planet with terrestrial composition depends on whether the orbit remains confined long enough to the habitable zone.

\section{The habitable zone}

All life on Earth requires liquid water during at least part of its life cycle. Consequently, it is usual to define the habitable zone (HZ) as the range of distances from a star within which any water at the surface of a terrestrial planet would be in the liquid phase (Kasting et al. 1993). A variety of criteria have been used to define the boundaries of the HZ. For the inner boundary we use the maximum distance from the star where a runaway greenhouse effect occurs leading to the evaporation of all surface water. For the outer boundary we use the maximum distance at which a cloud-free $\mathrm{CO}_{2}$ atmosphere can maintain a surface temperature of $273 \mathrm{~K}$. Kasting et al. (1993) have used these criteria in conjunction with climate models to obtain values for the boundary distances for various stars, and we have used these values. The values are conservative because of simplifying features in the models. Notably, at the inner boundary $\mathrm{H}_{2} \mathrm{O}$ cloud formation is neglected, which would cause this boundary to move inwards. At the outer boundary $\mathrm{CO}_{2}$ cloud formation is neglected. Forget \& Pierrehumbert (1997) have shown that the net effect of the formation of $\mathrm{CO}_{2}$ clouds is to warm the surface, through a scattering greenhouse effect from the small particles that make up the cloud, and this moves the outer boundary of the HZ outwards.

For zero-age main-sequence stars (ZAMS stars) the boundaries of the $\mathrm{HZ}$ lie closer to the star the later its spectral type. This is because of the combined effects of the star's lower luminosity and the shift in its spectrum to longer wavelengths. As a star ages, its luminosity and temperature increase and the inner boundary moves outwards. The outer boundary also moves outwards unless "cold starts" are prohibited, in which case it remains fixed at the ZAMS value. The estimated age of $47 \mathrm{UMa}$ is $7000 \mathrm{Ma}\left(1 \mathrm{Ma}=10^{6}\right.$ years) (Gonzalez 1998), and so the outward movement of the boundaries will have been significant.
There is however considerable overlap between the present and ZAMS HZs (Sect. 4), and so if terrestrial planets have survived in the $\mathrm{HZ}$ to the present time there is some prospect of finding a highly evolved biosphere.

\section{The method of orbital investigation}

To establish whether the orbit of an Earth-mass planet could remain confined to the $\mathrm{HZ}$ of $47 \mathrm{UMa}$ we must investigate the evolution of orbits launched in the HZ. Analytical integration is restricted to three bodies in (near) coplanar, (near) circular orbits. By contrast, the giants are in eccentric orbits, the Earthmass body would acquire an eccentric orbit, and we have at least four bodies not necessarily in coplanar orbits. We therefore use numerical integration. In particular we use the secondorder mixed-variable symplectic (MVS) integrator contained within the Mercury integrator package (Chambers 1999). This integrator originated with Levison \& Duncan (1994). It has since been extensively tested (Jones et al. 2001). MVS integrators are about ten times faster than other integrators when, as in exosystems, one body (the star) is the dominant gravitational influence. The symplectic property is that there is no build-up of errors in the total energy and total angular momentum of the system. The fractional errors were output in all our integrations and were always less than $10^{-6}$.

MVS integrators cannot handle close encounters between planets accurately, because the part of the Hamiltonian that describes the planetary interaction is then comparable with the star-planet Hamiltonian. The smallest distance at which it is safe to use the integrator is about three times the Hill radius of the planet in the encounter with the larger Hill radius. The Hill radius $R_{\mathrm{H}}$ is defined as

$R_{\mathrm{H}}=\left(\frac{m}{3 M_{*}}\right)^{1 / 3} a$

where $m$ is the mass of the planet, $a$ is its orbital semimajor axes, and $M_{*}$ is the mass of the star. When the two planets are separated by $R_{\mathrm{H}}$ their gravitational interaction is of the same order as the gravitational interaction of each planet with the star, and so considerable orbital modification will occur, particularly for the Earth-mass planet in an encounter with a giant. We halt integration at $3 R_{\mathrm{H}}$. This avoids using the MVS integrator in an inaccurate domain, and is also the point at which the eccentricity of the less massive planet will have become large, with a high probability of ejection or collision.

If the $>3 R_{\mathrm{H}}$ criterion is met throughout an integration then we classify the orbit as stable. But this alone is not sufficient. 
We also require that the orbit remains confined to the HZ, otherwise it is unlikely that life could evolve. We take confinement to mean that the semimajor axis remains in the $\mathrm{HZ}$ at all times in an integration that is not halted by a close encounter. An even tighter criterion would additionally restrict the orbits in the HZ to some upper limit of eccentricity, but we have not adopted such a criterion.

In setting the mass of the putative planet we take note of the result in Jones et al. (2001) that the outcome is not very sensitive to this mass. It is therefore fixed, and a value equal to the mass of the Earth plus the Moon has been used. The planet is accordingly labelled EM. Jones et al. (2001) also showed that the presence of a second Earth-mass planet affected the outcome not primarily through the direct gravitational interaction between these two planets, but through close encounters between them resulting from the effect of the giants on each of their orbits separately. We therefore restrict ourselves here to one planet, EM.

To start an integration we launch EM in an orbit with the small eccentricity $e_{\mathrm{EM}}=10^{-5}$. This is non-zero so that the longitude of the periastron, $\varpi_{\mathrm{EM}}$, is defined at $t=0$. For all three planets, at $t=0$, the longitudes of the ascending node and the mean anomalies are each set to zero. (Note that with zero inclination $i$ the longitude of the ascending node is undefined, but the input file requires some value, and we choose zero.) In most cases, at $t=0$, the inclination $i_{\mathrm{EM}}$ is zero with respect to the plane of the giants' orbits, which are assumed to be coplanar. The longitudes of the periastrons of the three planets are given various values.

We then integrate for a simulated time of $1000 \mathrm{Ma}$, unless the integration is halted automatically by a close encounter $\left(<3 R_{\mathrm{H}}\right)$. Ideally we would have liked to integrate for $7000 \mathrm{Ma}$, the estimated age of $47 \mathrm{UMa}$. However, in order to avoid integration inaccuracies, the integration time-step needs to be less than one-twentieth of the orbital period of the planet with the shortest period. For some configurations a single integration for 1000 Ma would then consume the order of a thousand hours of CPU time on the Compaq Alpha-based workstations used. Therefore, in order to explore a reasonable range of orbital parameters we had to set the integration for $1000 \mathrm{Ma}$. It is reassuring that only in a small minority of our integrations was there a close encounter after $500 \mathrm{Ma}$, and so there is a high probability that an orbit remaining confined for longer than 500 Ma will remain confined well beyond $1000 \mathrm{Ma}$.

Two sets of integrations have been performed with EM present. In the one set the giants have minimum mass, corresponding to $i_{0}=90^{\circ}$, and in the other they have 1.5 times the minimum, corresponding to $i_{0}=42^{\circ}$, which is a representative value (Sect. 1). In a third set of integrations there is no EM. Our purpose here is to investigate the eccentricity of the outer giant's orbit, which is constrained by the observations to $e_{\mathrm{G} 2}$ less than about 0.2 in the present epoch.

\section{Results}

Details of the 47 UMa system are given in Table 1. Figure 1 shows this system and also the 47 UMa system when it was thought to contain only one giant. The ZAMS habitable zone
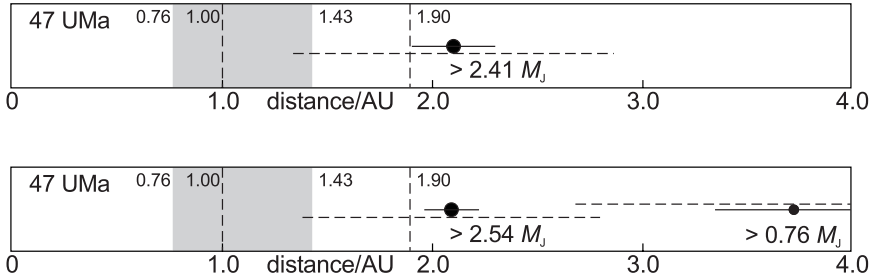

Fig. 1. The 47 Ursae Majoris system with one giant and with two giants (black circles). The shaded region is the habitable zone at zero age (main sequence), and the vertical lines are the boundaries of the present day habitable zone. See Sect. 4 for further details.

$\mathrm{HZ}(0)$ is included (shaded), and also the boundaries of the habitable zone today HZ(now) (vertical dashed lines). At each giant (black discs) the solid line shows the total excursion $2 \Delta r$ of the giant planet due to its eccentricity, and the dashed line extends to $\left(3 R_{\mathrm{H}}+\Delta r\right)$ each side of the giant for the case when the giant has its minimum mass. At 1.5 times the minimum mass $3 R_{\mathrm{H}}$ is increased by the small factor $1.5^{1 / 3}(=1.14)$ in accord with Eq. (1). In the integrations the changes in $a$ of the giants are small, typically $2-3 \%$ for the outer giant (G2) if there is no close encounter between the giants, otherwise about $10 \%$, and about $1 \%$ for the inner giant (G1).

\subsection{The eccentricity of the outer giant's orbit}

The goal here is to establish an upper limit on the outer giant's eccentricity $e_{\mathrm{G} 2}$ valid at the present epoch. Current observations set the upper limit for the present epoch at about 0.2. A value of 0.1 has been used in Fig. 1. Table 2 summarises a selection of our results with EM absent. In all runs

- $M_{47 \mathrm{UMa}}=1.03 M_{\odot}$

- at $t=0$

$$
\text { - } a_{\mathrm{G} 1}(0)=2.09 \mathrm{AU}, a_{\mathrm{G} 2}(0)=3.73 \mathrm{AU}, i_{\mathrm{G} 1}(0)=0 \text {, and }
$$
both mean anomalies are also zero

- the longitudes of the ascending node are also set to zero, though these longitudes are undefined except when $i_{\mathrm{G} 2}(0) \neq 0$.

Columns 2-6 specify the values at $t=0$ for all input parameters that are varied. Columns 7-11 summarise the outcome, where the encounter is the time into the integration when there was a close approach between the giants (G1 within $3 R_{\mathrm{H}}$ of the outer giant, in accord with Eq. (1)). The integration was halted at the first such encounter. In a few cases EM was included (not shown), which has demonstrated that no significantly different outcome for the evolution of the giants' orbits occurs when EM is present, provided that EM suffers no close encounters.

Consider first the case of 1.5 times the minimum giant masses. When both eccentricities at $t=0$ are set to $10^{-5}$ (row 1 in Table 2), then during the $1000 \mathrm{Ma}$ of the integration $e_{\mathrm{G} 2}(t)$ varies from close to zero to 0.024 , and $e_{\mathrm{G} 1}(t)$ varies from close to zero to a maximum of 0.011 . This maximum is considerably less than the currently observed value of 0.061 . Moreover there are no secular trends in $e_{\mathrm{G} 1}(t)$ and $e_{\mathrm{G} 2}(t)$ over the integration that could indicate larger eccentricities at $7000 \mathrm{Ma}$. Therefore, this particular simulation does not correspond to the 
Table 2. Orbital integrations for 47 UMa with two giants present (no terrestrial planets).

\begin{tabular}{|c|c|c|c|c|c|c|c|c|c|c|}
\hline \multicolumn{6}{|c|}{ input at $t=0$} & \multicolumn{5}{|c|}{ outcome } \\
\hline row & $e_{\mathrm{G} 1}$ & $e_{\mathrm{G} 2}$ & $\varpi_{\mathrm{G} 1}$ & $\varpi_{\mathrm{G} 2}$ & $i_{G 2}$ & enc'nter & range & range & range & range \\
\hline no. & & & $1^{\circ}$ & $1^{\circ}$ & $1^{\circ}$ & $/ \mathrm{Ma}$ & $a_{\mathrm{Gl}} / \mathrm{AU}$ & $a_{\mathrm{G} 2} / \mathrm{AU}$ & $e_{\mathrm{G} 1}$ & $e_{\mathrm{G} 2}$ \\
\hline \multicolumn{11}{|c|}{1.5 times minimum giant mass } \\
\hline$\overline{1}$ & $10^{-5}$ & $10^{-5}$ & 180 & 180 & 0 & $>1000$ & $2.086-2.090$ & $3.64-3.74$ & $10^{-5}-0.011$ & $10^{-5}-0.024$ \\
\hline 2 & 0.061 & $10^{-5}$ & 180 & 180 & 0 & $>1000$ & $2.087-2.093$ & $3.62-3.73$ & $0.0086-0.061$ & $10^{-5}-0.104$ \\
\hline 3 & 0.061 & $10^{-5}$ & 120 & 240 & 0 & $>1000$ & $2.088-2.094$ & $3.70-3.81$ & $0.021-0.105$ & $10^{-5}-0.105$ \\
\hline 4 & 0.061 & 0.10 & 180 & 180 & 0 & $>1000$ & $2.085-2.091$ & $3.62-3.74$ & $0.053-0.075$ & $0.047-0.105$ \\
\hline 5 & 0.061 & 0.10 & 180 & 180 & 10 & $>1000$ & $2.085-2.090$ & $3.62-3.74$ & $0.053-0.076$ & 0.049-0.104 \\
\hline 6 & 0.061 & 0.10 & 120 & 240 & 0 & $>1000$ & $2.086-2.097$ & $3.69-3.83$ & $5 \times 10^{-5}-0.083$ & $0.051-0.163$ \\
\hline 7 & 0.061 & 0.10 & 120 & 240 & 10 & $>1000$ & $2.087-2.096$ & $3.69-3.82$ & $0.0012-0.083$ & $0.047-0.158$ \\
\hline 8 & 0.061 & 0.10 & 120 & 230 & 0 & $>1000$ & $2.088-2.097$ & $3.68-3.81$ & $0.0067-0.085$ & $0.037-0.156$ \\
\hline 9 & 0.061 & 0.12 & 180 & 180 & 0 & $>1000$ & $2.083-2.090$ & $3.63-3.76$ & $0.037-0.085$ & $0.0089-0.136$ \\
\hline 10 & 0.061 & 0.12 & 180 & 180 & 10 & 2.58 & $2.067-2.092$ & $3.62-4.04$ & $0.0058-0.110$ & $0.0022-0.257$ \\
\hline 11 & 0.061 & 0.12 & 120 & 240 & 0 & 2.21 & $2.078-2.097$ & $3.68-4.03$ & $0.0016-0.112$ & $0.085-0.242$ \\
\hline 12 & 0.061 & 0.12 & 120 & 240 & 10 & 161 & $2.067-2.097$ & $3.69-4.18$ & $0.00071-0.109$ & $0.030-0.291$ \\
\hline 13 & 0.061 & 0.12 & 120 & 230 & 0 & $>1000$ & $2.087-2.098$ & $3.68-3.82$ & $0.0050-0.092$ & $0.052-0.175$ \\
\hline \multicolumn{11}{|c|}{ minimum giant mass } \\
\hline 1 & 0.061 & 0.12 & 180 & 180 & 0 & $>1000$ & $2.086-2.090$ & $3.66-3.74$ & $0.056-0.093$ & $0.026-0.127$ \\
\hline 2 & 0.061 & 0.12 & 180 & 180 & 10 & $>1000$ & $2.086-2.090$ & $3.66-3.74$ & $0.056-0.095$ & $0.026-0.127$ \\
\hline 3 & 0.061 & 0.12 & 120 & 240 & 0 & $>1000$ & $2.088-2.095$ & $3.70-3.80$ & $6.2 \times 10^{-5}-0.086$ & $0.073-0.172$ \\
\hline 4 & 0.061 & 0.12 & 120 & 240 & 10 & $>1000$ & $2.088-2.095$ & $3.70-3.80$ & $5.5 \times 10^{-5}-0.086$ & $0.068-0.166$ \\
\hline 5 & 0.061 & 0.12 & 120 & 230 & 0 & $>1000$ & $2.088-2.095$ & $3.70-3.79$ & $0.0063-0.089$ & $0.060-0.166$ \\
\hline 6 & 0.061 & 0.15 & 180 & 180 & 0 & $>1000$ & $2.084-2.090$ & $3.66-3.75$ & $0.056-0.111$ & $0.0066-0.158$ \\
\hline 7 & 0.061 & 0.15 & 180 & 180 & 10 & $>1000$ & $2.084-2.090$ & $3.66-3.75$ & $0.043-0.113$ & $0.0031-0.160$ \\
\hline 8 & 0.061 & 0.15 & 120 & 240 & 0 & 318 & $2.056-2.096$ & $3.69-4.28$ & $0.00020-0.097$ & $0.090-0.321$ \\
\hline 9 & 0.061 & 0.15 & 120 & 240 & 10 & $>1000$ & $2.086-2.095$ & $3.70-3.81$ & $0.00050-0.126$ & $0.044-0.203$ \\
\hline 10 & 0.061 & 0.15 & 120 & 230 & 0 & $>1000$ & $2.087-2.096$ & $3.69-3.80$ & 0.0034-0.099 & $0.085-0.196$ \\
\hline 11 & 0.061 & 0.17 & 180 & 180 & 0 & $>1000$ & $2.083-2.091$ & $3.67-3.76$ & $0.056-0.124$ & $1.2 \times 10^{-5}-0.180$ \\
\hline 12 & 0.061 & 0.17 & 180 & 180 & 10 & $>1000$ & $2.083-2.090$ & $3.66-3.76$ & $0.055-0.126$ & $1.1 \times 10^{-5}-0.183$ \\
\hline 13 & 0.061 & 0.17 & 120 & 240 & 0 & 0.0082 & $2.064-2.090$ & $3.73-4.17$ & $0.043-0.061$ & $0.170-0.311$ \\
\hline 14 & 0.061 & 0.17 & 120 & 240 & 10 & 0.510 & $2.050-2.094$ & $3.71-4.36$ & $0.0058-0.134$ & $0.124-0.334$ \\
\hline 15 & 0.061 & 0.17 & 120 & 230 & 0 & 0.294 & $2.065-2.092$ & $3.70-4.16$ & $0.024-0.106$ & $0.114-0.309$ \\
\hline 16 & 0.061 & 0.17 & 172 & 127 & 0 & 0.833 & $2.075-2.096$ & $3.63-3.97$ & $0.012-0.118$ & $0.012-0.252$ \\
\hline
\end{tabular}

observations. This is because the planets have been launched with too much angular momentum (which is proportional to $\left.\left(1-e^{2}\right)^{1 / 2}\right)$. Conservation of angular momentum then ensures that large values of $e$ cannot be acquired.

In the remaining rows in Table 2 are some results when $\mathrm{G} 1$ is launched with its presently observed value of $e_{\mathrm{G} 1}=$ 0.061 , and so we are investigating the evolution of the system from any time at which $e_{\mathrm{G} 1}$ has the value 0.061 up to $1000 \mathrm{Ma}$ beyond that time. In row $2 \mathrm{G} 2$ is launched with $e_{\mathrm{G} 2}(0)=10^{-5}$. This subsequently varies between $10^{-5}$ and 0.104 during the $1000 \mathrm{Ma}$ run. In row 3 the starting configuration differs from that in row 2 only in that the values of the longitude of the periastron, $\varpi_{\mathrm{G} 1}(0)$ and $\varpi_{\mathrm{G} 2}(0)$, are now $120^{\circ}$ and $240^{\circ}$ respectively, instead of $180^{\circ}$ in each case in row 2 . The differences $\Delta \varpi_{21}(0)=\left(\varpi_{\mathrm{G} 2}(0)-\varpi_{\mathrm{G} 1}(0)\right)$ are $120^{\circ}$ and $0^{\circ}$ respectively. These configurations are shown in Fig. 2, where the arrows indicate the periastrons. The currently observed difference is $45^{\circ}$, but with large uncertainties so that the actual current value is likely, at a one standard deviation level, to be between about $0^{\circ}$ and $120^{\circ}$ (Fischer et al. 2002). The outcome in row 3 is broadly similar to that in row 2 but is not identical. This shows sensitivity to the starting positions of the planets in their orbits. We will return to this shortly. In the next five rows the value of $e_{\mathrm{G} 2}(0)=0.10$. For each of $\Delta \varpi_{21}(0)=0^{\circ}$ and $120^{\circ}$ there are pairs of runs at $i_{\mathrm{G} 2}(0)=0$ and $10^{\circ}$. A fifth run at $\Delta \varpi_{21}(0)=110^{\circ}$ has $i_{\mathrm{G} 2}(0)=0^{\circ}$. In all five cases there were no close encounters, and so the integration ran for the full $1000 \mathrm{Ma}$ though the details of the outcomes differ, particularly in the range of $e_{\mathrm{G} 2}(t)$ in the final column of Table 2.

The next five rows repeat the launch configurations of the previous five, except that $e_{\mathrm{G} 2}(0)=0.12$. Here, three of the five result in G1-G2 encounters that end the runs well short 


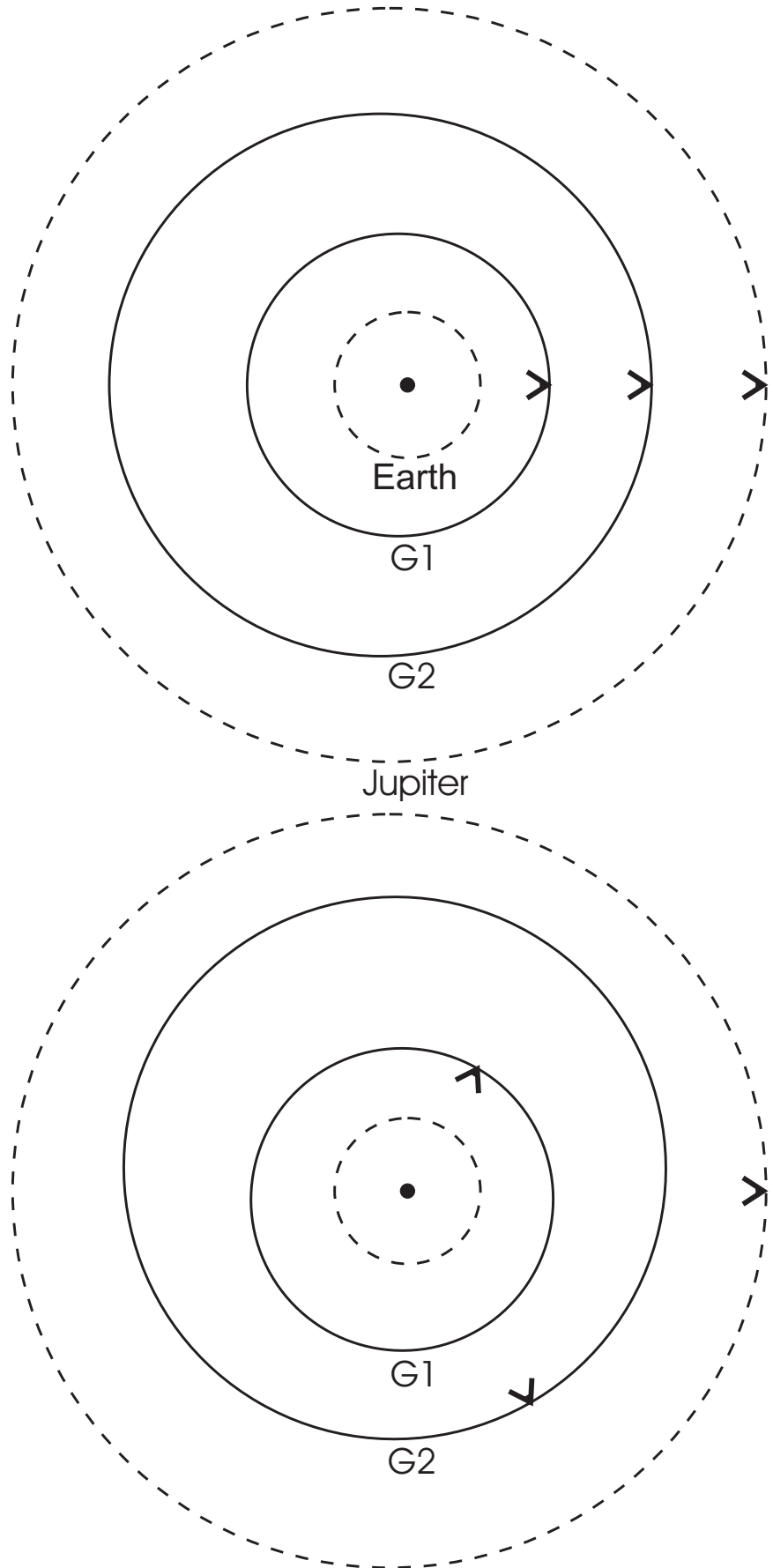

Fig. 2. Two configurations of 47 Ursae Majoris at $t=0$. In the upper configuration the longitudes of the periastrons of both giants is $180^{\circ}$, and in the lower configuration that of the inner giant (G1) is $120^{\circ}$ and the outer giant $(\mathrm{G} 2) 240^{\circ}$.

of $1000 \mathrm{Ma}$, and Fig. 3 shows the case in row 11 (note that the orbital elements have been sampled every 10000 years, plus the terminal values). It is not surprising that an increase in $e_{\mathrm{G} 2}(0)$ from 0.10 to 0.12 has led to early encounters. What does need a bit of investigation is why, at a given value of $e_{\mathrm{G} 2}(0)$, some launch configurations lead to an early close encounter whilst others do not. Figure 4 illustrates why this is so. Figure 4a shows $\Delta \varpi_{21}(t)$ for $\Delta \varpi_{21}(0)=0^{\circ}$ (row 9). The value librates around zero nearly all the time (the small gaps are
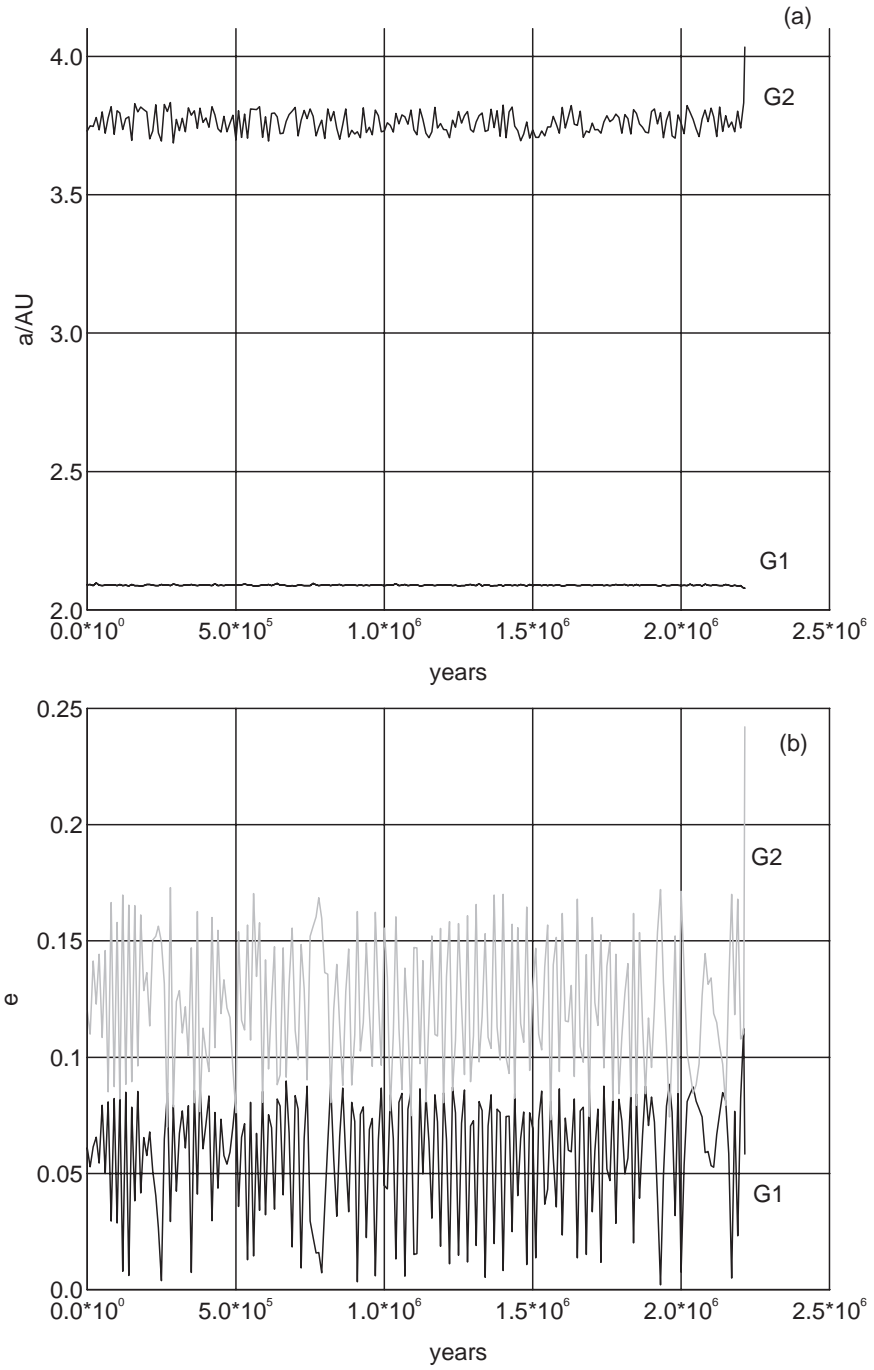

Fig. 3. The semimajor axis and eccentricity of G1 and G2 ending in a close encounter between them. This is for row 11 in Table 2. The orbital elements have been sampled every 10000 years, plus the terminal values.

values near $\pm 180^{\circ}$ ), and so close encounters between the giants are avoided. Figure $4 \mathrm{~b}$ shows $\Delta \varpi_{21}(t)$ for $\Delta \varpi_{21}(0)=120^{\circ}$ (row 11). The value now circulates and so close encounters between the giants occur periodically, and this has led to the early close encounter. Laughlin et al. (2002) have applied secular theory to the $47 \mathrm{UMa}$ system, and have shown that at $e_{\mathrm{G} 2}(0)=0.12$, if $\Delta \varpi_{21}(0)=0^{\circ}, \Delta \varpi_{21}(t)$ will librate around $0^{\circ}$, and will circulate if $\Delta \varpi_{21}(0)=120^{\circ}$. (Note: secular theory shows that whether there is libration or circulation depends on $\left|\Delta \varpi_{21}(0)\right|$ so the sign of $\Delta \varpi_{21}(0)$ is immaterial, a conclusion we have demonstrated by reversing the $\varpi_{\mathrm{G} 2}(0)$ and $\varpi_{\mathrm{G} 1}(0)$ values.) In row $10 \Delta \varpi_{21}(0)=0^{\circ}$ but there is an early encounter. In this case G2 has been launched with an inclination of $10^{\circ}$, and this has caused the libration around zero to break down $-\Delta \varpi_{21}(t)$ displays periods of libration, periods of circulation, and periods of anti-libration.

We take the conservative line that if, for a given value of $e_{\mathrm{G} 2}(0)$, any launch configuration leads to a close encounter within $1000 \mathrm{Ma}$, then we will not look among this group of 

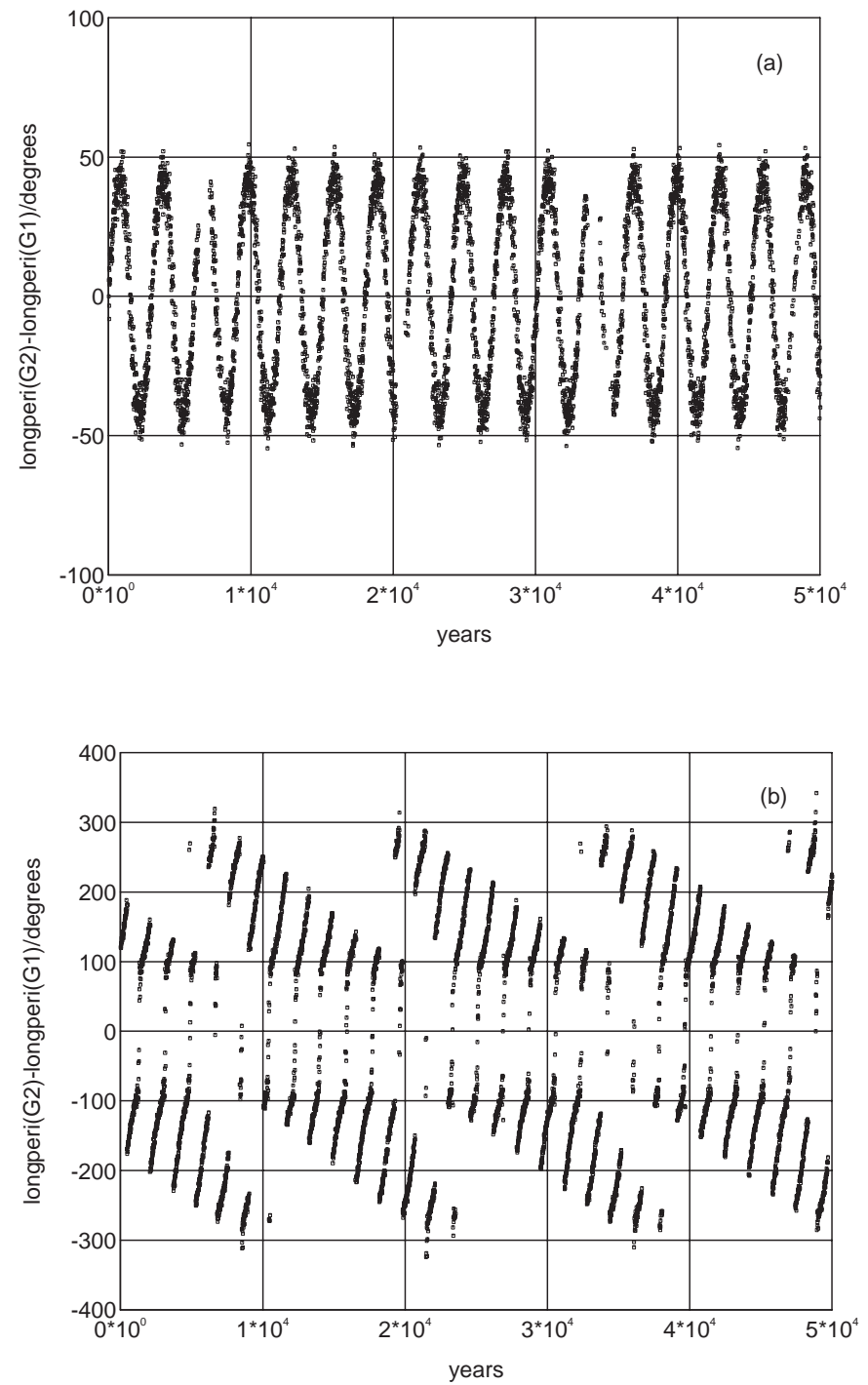

Fig. 4. For G1 and G2 a) libration, associated with relative stability (row 9 in Table 2) b) circulation, associated with relative instability (row 11 in Table 2).

runs for the maximum value that $e_{\mathrm{G} 2}(t)$ might have today. We thus disregard $e_{\mathrm{G} 2}(0)=0.12$. For values of $e_{\mathrm{G} 2}(0)$ of $0.13,0.15$, and 0.20 (not shown) we get early termination for most or all of the starting configurations, so these runs are also disregarded.

We thus conclude that $e_{\mathrm{G} 2}(0)=0.10$ (rows $4-8$ in Table 2) is the group of runs where we will obtain a good estimate of the upper limit of $e_{\mathrm{G} 2}$ at the present time. One approach is to look for the maximum value of $e_{\mathrm{G} 2}(t)$ that occurs at any time in these runs, and you can see from Table 2 that this is 0.163 . However, because of the conservation of angular momentum, there is a strong anticorrelation between $e_{\mathrm{G} 1}(t)$ and $e_{\mathrm{G} 2}(t)$. This is apparent in Fig. 3, where the eccentricities have been sampled every 10000 years. Because of this anticorrelation, a better approach is to look for the maximum value of $e_{\mathrm{G} 2}(t)$ when at the same time $e_{\mathrm{Gl}}(t)$ has its present value of 0.061 . In rows $4-8$ these maxima are respectively $0.100,0.102,0.127,0.119$, and 0.118 , and so the limit is about 0.13 . Finally, and over-conservatively, the value of 0.10 could itself be taken as the upper limit.
Table 2 also shows some results at the minimum giant masses. By following the same procedure as for 1.5 times the minimum giant masses, we settle on $e_{\mathrm{G} 2}(0)=0.12$ and obtain the three upper limits 0.172 (optimistic), 0.15 , and (overconservatively) 0.12 .

Note that at 1.5 times the minimum mass in Table 2 there is just one case where the orbit became unstable later than $100 \mathrm{Ma}$. Among the 9 runs at $e_{\mathrm{G} 2}(0)$ of $0.13,0.15$, and 0.20 , there was just one further case. The proportion is similar at minimum mass. Orbits that last for $1000 \mathrm{Ma}$ are thus likely to last for very much longer.

\subsection{The fate of EM at 1.5 times minimum giant masses}

With EM now included, Tables $3 a$ and $b$ show the results of our orbital integrations at 1.5 times the minimum giant masses, corresponding to $i_{0}=42^{\circ}$, which is a reasonable estimate of the inclination (Gonzalez 1998; Pourbaix 2001; Perryman et al. 1996). In all runs

- $M_{47 \mathrm{UMa}}=1.03 M_{\odot}$ (as before);

- at $t=0$, for the giants, $a_{\mathrm{G} 1}(0)=2.09 \mathrm{AU}, e_{\mathrm{G} 1}(0)=0.061$, $a_{\mathrm{G} 2}(0)=3.73 \mathrm{AU}, i_{\mathrm{G} 1}(0)=0$, and the mean anomalies and longitudes of the ascending node are also zero;

- at $t=0$, for EM, $e_{\mathrm{EM}}(0)=10^{-5}$, and the mean anomaly and longitude of the ascending node are zero.

Columns $2-7$ specify the values at $t=0$ for all input parameters that are varied. The remaining columns summarise the outcome, where the encounter is the time into the integration when there was a close approach (within $3 R_{\mathrm{H}}$ ) between EM and a giant, which was G1 in every case. The integration was halted at the first such encounter.

More runs were done at $a_{\mathrm{EM}}(0)=1.05 \mathrm{AU}$ than at any other value (Table 3a). A value of $1.05 \mathrm{AU}$ is in $\mathrm{HZ}$ (now), just within its inner boundary, yet lies away from the mean motion resonances. The more telling set of runs is with $e_{\mathrm{G} 2}(0)=0.1$ (rows 25-30 and 32-35), close to the maximum value and therefore likely to discriminate sensitively between different launch configurations. First, note that whether $\varpi_{\mathrm{EM}}(0)=0^{\circ}$ or $180^{\circ}$ does not make a lot of difference, and this is true nearly everywhere in Tables $3 a$ and $b$. This is unsurprising given that $e_{\mathrm{EM}}(0)$ is only $10^{-5}$. That there is any difference at all is a measure of the sensitivity of the outcome to the starting values. This could be further studied at mean motion resonances.

Second, note that runs with $\left|\Delta \varpi_{21}(0)\right|=90^{\circ}$ last for $>1000 \mathrm{Ma}$ whereas that with $\left|\Delta \varpi_{21}(0)\right|=0^{\circ}$ lasts for only $5.79 \mathrm{Ma}$, and $\left|\Delta \varpi_{21}(0)\right|=120^{\circ}$ for only $2.27 \mathrm{Ma}$. By contrast $\left|\Delta \varpi_{21}(0)\right|=110^{\circ}$ and $130^{\circ}$ also run for $>1000 \mathrm{Ma}$. This indicates that $\left|\Delta \varpi_{21}(0)\right|=90^{\circ}$ is a comparatively stable launch configuration, and that $\left|\Delta \varpi_{21}(0)\right|=120^{\circ}$ is comparatively unstable. Examination of the rest of these tables confirms this impression. We have not seen any convincing evidence of libration of $\Delta \varpi_{\mathrm{EM} 1}(t)$ and $\Delta \varpi_{\mathrm{EM} 2}(t)$ around $0^{\circ}$, and so libration versus circulation has not been established as the difference between the stable and unstable cases. But we have seen a difference in the degree of $e_{\mathrm{EM}}-\Delta \varpi_{\mathrm{EM} 1}$ coupling, as illustrated in Fig. 5a for 
Table 3a. Orbital integrations for 47 UMa with two giants present, 1.5 times minimum mass.

\begin{tabular}{|c|c|c|c|c|c|c|c|c|c|c|}
\hline \multicolumn{7}{|c|}{ input at $t=0$} & \multicolumn{4}{|c|}{ outcome } \\
\hline row & $a_{\mathrm{EM}}$ & $i_{\mathrm{EM}}$ & $e_{\mathrm{G} 2}$ & $\varpi_{\mathrm{EM}}$ & $\varpi_{\mathrm{G} 1}$ & $\varpi_{\mathrm{G} 2}$ & enc'nter & range & range & range \\
\hline no. & /AU & $1^{\circ}$ & $1^{\circ}$ & $1^{\circ}$ & $1^{\circ}$ & $1^{\circ}$ & $/ \mathrm{Ma}$ & $a_{\mathrm{EM}} / \mathrm{AU}$ & $e_{\mathrm{EM}}$ & $i_{\mathrm{EM}} /^{\circ}$ \\
\hline 1 & 1.40 & 0 & $10^{-5}$ & 0 & 120 & 240 & 8.4 years & $1.400-1.415$ & $10^{-5}-0.070$ & \\
\hline 2 & 1.40 & 0 & 0.1 & 0 & 120 & 240 & 8.4 years & $1.400-1.414$ & $10^{-5}-0.072$ & \\
\hline 3 & 1.25 & 0 & $10^{-5}$ & 0 & 180 & 180 & $>1000$ & $1.241-1.257$ & $10^{-5}-0.069$ & \\
\hline 4 & 1.25 & 0 & $10^{-5}$ & 0 & 120 & 240 & 4.52 & $1.239-1.270$ & $10^{-5}-0.121$ & \\
\hline 5 & 1.25 & 0 & 0.1 & 0 & 180 & 90 & 483 & $1.240-1.271$ & $10^{-5}-0.107$ & \\
\hline 6 & 1.25 & 0 & 0.1 & 0 & 180 & 180 & 0.936 & $1.239-1.267$ & $10^{-5}-0.114$ & \\
\hline 7 & 1.25 & 0 & 0.1 & 0 & 120 & 240 & 0.050 & $1.236-1.269$ & $10^{-5}-0.147$ & \\
\hline 8 & 1.20 & 0 & $10^{-5}$ & 0 & 180 & 90 & 918 & $1.192-1.218$ & $10^{-5}-0.187$ & \\
\hline 9 & 1.20 & 0 & $10^{-5}$ & 0 & 120 & 240 & 634 & $1.195-1.217$ & $10^{-5}-0.177$ & \\
\hline 10 & 1.20 & 0 & 0.1 & 0 & 180 & 90 & 3.86 & $1.193-1.213$ & $10^{-5}-0.161$ & \\
\hline 11 & 1.20 & 0 & 0.1 & 180 & 180 & 90 & 0.227 & $1.178-1.200$ & $10^{-5}-0.189$ & \\
\hline 12 & 1.15 & 0 & $10^{-5}$ & 0 & 180 & 180 & $>1000$ & $1.146-1.155$ & $10^{-5}-0.096$ & \\
\hline 13 & 1.15 & 0 & $10^{-5}$ & 0 & 120 & 240 & $>1000$ & $1.148-1.157$ & $10^{-5}-0.107$ & \\
\hline 14 & 1.15 & 0 & 0.1 & 0 & 180 & 90 & 319 & $1.143-1.160$ & $10^{-5}-0.234$ & \\
\hline 15 & 1.15 & 0 & 0.1 & 0 & 180 & 180 & 83 & $1.145-1.166$ & $10^{-5}-0.200$ & \\
\hline 16 & 1.15 & 0 & 0.1 & 0 & 120 & 240 & 1.12 & $1.138-1.158$ & $10^{-5}-0.234$ & \\
\hline 17 & 1.10 & 0 & $10^{-5}$ & 0 & 120 & 240 & $>1000$ & $1.098-1.106$ & $10^{-5}-0.109$ & \\
\hline 18 & 1.10 & 0 & 0.1 & 0 & 180 & 90 & $>1000$ & $1.096-1.105$ & $10^{-5}-0.177$ & \\
\hline 19 & 1.10 & 0 & 0.1 & 180 & 180 & 90 & 833 & $1.090-1.104$ & $10^{-5}-0.259$ & \\
\hline 20 & 1.10 & 0 & 0.1 & 0 & 120 & 240 & 3.49 & $1.098-1.110$ & $10^{-5}-0.319$ & \\
\hline 21 & 1.10 & 0 & 0.1 & 0 & 120 & 230 & $>1000$ & $1.096-1.110$ & $10^{-5}-0.268$ & \\
\hline 22 & 1.10 & 0 & 0.1 & 0 & 110 & 240 & 896 & $1.079-1.108$ & $10^{-5}-0.457$ & \\
\hline 23 & 1.10 & 0 & 0.1 & 0 & 100 & 240 & 3.43 & $1.006-1.113$ & $10^{-5}-0.313$ & \\
\hline 24 & 1.05 & 0 & $10^{-5}$ & 0 & 120 & 240 & $>1000$ & 1.049-1.054 & $10^{-5}-0.118$ & \\
\hline 25 & 1.05 & 0 & 0.1 & 0 & 180 & 90 & $>1000$ & $1.047-1.054$ & $10^{-5}-0.186$ & \\
\hline 26 & 1.05 & 0 & 0.1 & 180 & 180 & 90 & $>1000$ & $1.044-1.051$ & $10^{-5}-0.189$ & \\
\hline 27 & 1.05 & 0 & 0.1 & 0 & 180 & 180 & 5.79 & $1.045-1.054$ & $10^{-5}-0.292$ & \\
\hline 28 & 1.05 & 0 & 0.1 & 0 & 120 & 240 & 2.27 & $1.046-1.055$ & $10^{-5}-0.367$ & \\
\hline 29 & 1.05 & 0 & 0.1 & 0 & 120 & 230 & $>1000$ & $1.048-1.055$ & $10^{-5}-0.217$ & \\
\hline 30 & 1.05 & 0 & 0.1 & 0 & 110 & 240 & $>1000$ & $1.040-1.057$ & $10^{-5}-0.418$ & \\
\hline 31 & 1.05 & 10 & $10^{-5}$ & 0 & 120 & 240 & $>1000$ & $1.048-1.055$ & $10^{-5}-0.154$ & $9.7-10.2$ \\
\hline 32 & 1.05 & 10 & 0.1 & 0 & 180 & 90 & $>1000$ & $1.046-1.054$ & $10^{-5}-0.228$ & $9.6-10.6$ \\
\hline 33 & 1.05 & 10 & 0.1 & 180 & 180 & 90 & $>1000$ & $1.044-1.051$ & $10^{-5}-0.228$ & $9.6-10.5$ \\
\hline 34 & 1.05 & 10 & 0.1 & 0 & 180 & 180 & 1.88 & $1.043-1.054$ & $10^{-5}-0.361$ & $9.3-11.2$ \\
\hline 35 & 1.05 & 10 & 0.1 & 0 & 120 & 240 & 0.017 & $1.050-1.054$ & $10^{-5}-0.401$ & $9.9-11.6$ \\
\hline 36 & 1.00 & 0 & $10^{-5}$ & 0 & 180 & 90 & 0.753 & $0.994-1.213$ & $10^{-5}-0.691$ & \\
\hline 37 & 1.00 & 0 & $10^{-5}$ & 0 & 180 & 180 & 0.0079 & $0.983-1.020$ & $10^{-5}-0.726$ & \\
\hline 38 & 1.00 & 0 & 0.1 & 0 & 120 & 240 & 0.238 & $0.968-1.010$ & $10^{-5}-0.694$ & \\
\hline 39 & 1.00 & 0 & 0.1 & 0 & 120 & 230 & 0.144 & $0.991-1.010$ & $10^{-5}-0.555$ & \\
\hline
\end{tabular}

row 25 in Table 3a, and Fig. $5 \mathrm{~b}$ for row 28. For $\left|\Delta \varpi_{21}(0)\right|=90^{\circ}$ the coupling is strong, such that when $e_{\mathrm{EM}}$ is large $\Delta \varpi_{\mathrm{EM} 1}$ is close to $0^{\circ}$ (or equivalently to $\pm 360^{\circ}$ ), and so close encounters between EM and G1 are avoided. For $\left|\Delta \varpi_{21}(0)\right|=120^{\circ}$ the coupling is weaker, and so close encounters between EM and
G1 are less strongly avoided. This kind of coupling is known to protect some asteroids on planet-crossing orbits in the Solar System.

At $a_{\mathrm{EM}}(0)=1.05 \mathrm{AU}$ we also did some integrations with $i_{\mathrm{G} 1}(0)=10^{\circ}$ instead of $0^{\circ}$, shown in rows $31-35$ in Table $3 \mathrm{a}$. 
Table 3b. Orbital integrations for 47 UMa with two giants present, 1.5 times minimum mass.

\begin{tabular}{|c|c|c|c|c|c|c|c|c|c|}
\hline \multicolumn{7}{|c|}{ input at $t=0$} & \multicolumn{3}{|c|}{ outcome } \\
\hline row & $a_{\mathrm{EM}}$ & $i_{\mathrm{EM}}$ & $e_{\mathrm{G} 2}$ & $\varpi_{\mathrm{EM}}$ & $\varpi_{\mathrm{G} 1}$ & $\varpi_{\mathrm{G} 2}$ & enc'nter & range & range \\
\hline no. & /AU & $1^{\circ}$ & $1^{\circ}$ & $1^{\circ}$ & $1^{\circ}$ & $1^{\circ}$ & $/ \mathrm{Ma}$ & $a_{\mathrm{EM}} / \mathrm{AU}$ & $e_{\mathrm{EM}}$ \\
\hline 1 & 0.95 & 0 & $10^{-5}$ & 0 & 120 & 240 & $>1000$ & $0.949-0.953$ & $10^{-5}-0.157$ \\
\hline 2 & 0.95 & 0 & 0.1 & 0 & 180 & 90 & 0.110 & $0.947-1.001$ & $10^{-5}-0.587$ \\
\hline 3 & 0.95 & 0 & 0.1 & 180 & 180 & 90 & 0.117 & $0.948-0.975$ & $10^{-5}-0.466$ \\
\hline 4 & 0.95 & 0 & 0.1 & 0 & 180 & 180 & 10.0 & $0.952-0.974$ & $10^{-5}-0.465$ \\
\hline 5 & 0.95 & 0 & 0.1 & 0 & 120 & 230 & 0.091 & $0.950-0.955$ & $10^{-5}-0.542$ \\
\hline 6 & 0.90 & 0 & $10^{-5}$ & 0 & 180 & 180 & $>1000$ & $0.899-0.902$ & $10^{-5}-0.179$ \\
\hline 7 & 0.90 & 0 & $10^{-5}$ & 0 & 120 & 240 & 0.021 & $0.900-1.000$ & $10^{-5}-0.538$ \\
\hline 8 & 0.90 & 0 & 0.1 & 0 & 180 & 90 & $>1000$ & $0.898-0.903$ & $10^{-5}-0.224$ \\
\hline 9 & 0.90 & 0 & 0.1 & 180 & 180 & 90 & $>1000$ & $0.897-0.901$ & $10^{-5}-0.225$ \\
\hline 10 & 0.90 & 0 & 0.1 & 0 & 120 & 240 & 7.31 & $0.898-0.945$ & $10^{-5}-0.528$ \\
\hline 11 & 0.90 & 0 & 0.1 & 0 & 120 & 230 & $>1000$ & $0.900-0.903$ & $10^{-5}-0.178$ \\
\hline 12 & 0.85 & 0 & $10^{-5}$ & 0 & 120 & 240 & $>1000$ & $0.849-0.852$ & $10^{-5}-0.258$ \\
\hline 13 & 0.85 & 0 & 0.1 & 0 & 120 & 240 & 15.8 & $0.848-0.960$ & $10^{-5}-0.644$ \\
\hline 14 & 0.85 & 0 & 0.1 & 0 & 120 & 230 & $>1000$ & $0.850-0.852$ & $10^{-5}-0.118$ \\
\hline 15 & 0.80 & 0 & $10^{-5}$ & 0 & 120 & 240 & $>1000$ & $0.800-0.801$ & $10^{-5}-0.0616$ \\
\hline 16 & 0.80 & 0 & 0.1 & 0 & 120 & 240 & 106 & $0.794-0.947$ & $10^{-5}-0.658$ \\
\hline 17 & 0.80 & 0 & 0.1 & 0 & 120 & 230 & $>1000$ & $0.800-0.801$ & $10^{-5}-0.0910$ \\
\hline 18 & 0.76 & 0 & $10^{-5}$ & 0 & 180 & 180 & $>1000$ & $0.760-0.761$ & $10^{-5}-0.476$ \\
\hline 19 & 0.76 & 0 & $10^{-5}$ & 0 & 120 & 240 & $>1000$ & $0.760-0.761$ & $10^{-5}-0.0579$ \\
\hline 20 & 0.76 & 0 & 0.1 & 0 & 180 & 90 & $>1000$ & $0.760-0.761$ & $10^{-5}-0.0364$ \\
\hline 21 & 0.76 & 0 & 0.1 & 0 & 180 & 180 & 0.695 & $0.758-1.048$ & $10^{-5}-0.767$ \\
\hline 22 & 0.76 & 0 & 0.1 & 0 & 120 & 240 & 167 & $0.757-0.942$ & $10^{-5}-0.658$ \\
\hline 23 & 0.76 & 0 & 0.1 & 0 & 120 & 230 & $>1000$ & $0.760-0.761$ & $10^{-5}-0.0855$ \\
\hline 24 & 0.70 & 0 & $10^{-5}$ & 0 & 180 & 180 & $>1000$ & $0.6997-0.7005$ & $10^{-5}-0.058$ \\
\hline 25 & 0.70 & 0 & $10^{-5}$ & 0 & 120 & 240 & $>1000$ & $0.6999-0.7006$ & $10^{-5}-0.062$ \\
\hline 26 & 0.70 & 0 & 0.1 & 0 & 180 & 90 & $>1000$ & $0.6996-0.7005$ & $10^{-5}-0.096$ \\
\hline 27 & 0.70 & 0 & 0.1 & 0 & 180 & 180 & 1.00 & $0.700-1.101$ & $10^{-5}-0.776$ \\
\hline 28 & 0.70 & 0 & 0.1 & 0 & 120 & 240 & 157 & $0.695-1.007$ & $10^{-5}-0.722$ \\
\hline
\end{tabular}

You can see that the outcome is broadly the same as at $0^{\circ}$. Overall, launching EM at 1.05 AU meets with mixed fortunes - some launch configurations run for $>1000 \mathrm{Ma}$, whilst others end in close encounters at $<1 \mathrm{Ma}$.

The upper half of Fig. 6, labelled "1.5 min mass", summarises the results in Tables $3 \mathrm{a}$ and $\mathrm{b}$. The positions of the mean motion resonances between EM and G1 are indicated by the non-italicised period ratios along the top, and between $\mathrm{EM}$ and $\mathrm{G} 2$ by the italicised ratios. The habitable zones are shown, and also the inward extremity of $\left(3 R_{\mathrm{H}}+\Delta r\right)$ for $\mathrm{G} 1$ by the horizontal dashed line. A circle denotes a run that lasted for the full $1000 \mathrm{Ma}$, and a cross one that resulted in a close encounter within $1000 \mathrm{Ma}$. The mixed fortunes at $1.05 \mathrm{AU}$ for $e_{\mathrm{G} 2}(0)=0.1$ are signified by a cross and circle at this location. At $e_{\mathrm{G} 2}(0)=10^{-5}$ there is only a circle - Table $3 \mathrm{a}$ rows 24 and 31 show that the unstable launch configuration $\left|\Delta \varpi_{21}(0)\right|=120^{\circ}$ ran for $>1000 \mathrm{Ma}$ so it is likely that the other configurations would also run for $>1000 \mathrm{Ma}$.
Figure 6 shows that there are no stable orbits at $a_{\mathrm{EM}}(0)=$ 1.4 AU, surely because this is within $\left(3 R_{\mathrm{H}}+\Delta r\right)$ of G1. At $a_{\mathrm{EM}}(0)=1.25 \mathrm{AU} \mathrm{EM}$ starts not much further away, so the instability here is also not surprising. Otherwise, at 1.5 times the minimum giant masses, provided that we avoid low-order mean-motion resonances, particularly with G1, stable orbits can be found in the inner region of $\mathrm{HZ}$ (now) and in a greater proportion of $\mathrm{HZ}(0)$, particularly if $e_{\mathrm{G} 2}(0)<<0.1$.

It can be seen from Tables $3 \mathrm{a}$ and $\mathrm{b}$ that for the stable orbits the excursions in $a_{\mathrm{EM}}(t)$ are slight, and so $a_{\mathrm{EM}}(t)$ in stable cases always remains confined to the habitable zones. The eccentricities in a stable run range from close to zero to maxima typically as large as 0.2 , about twice that of the orbit of Mars. The apastrons remain in the habitable zone, but with $a_{\mathrm{EM}}(t)$ near the inner boundary of the habitable zone the periastron will be interior to this inner boundary some of the time. During such periods of comparatively large eccentricity, EM would be subject to large variations in stellar irradiance with large 

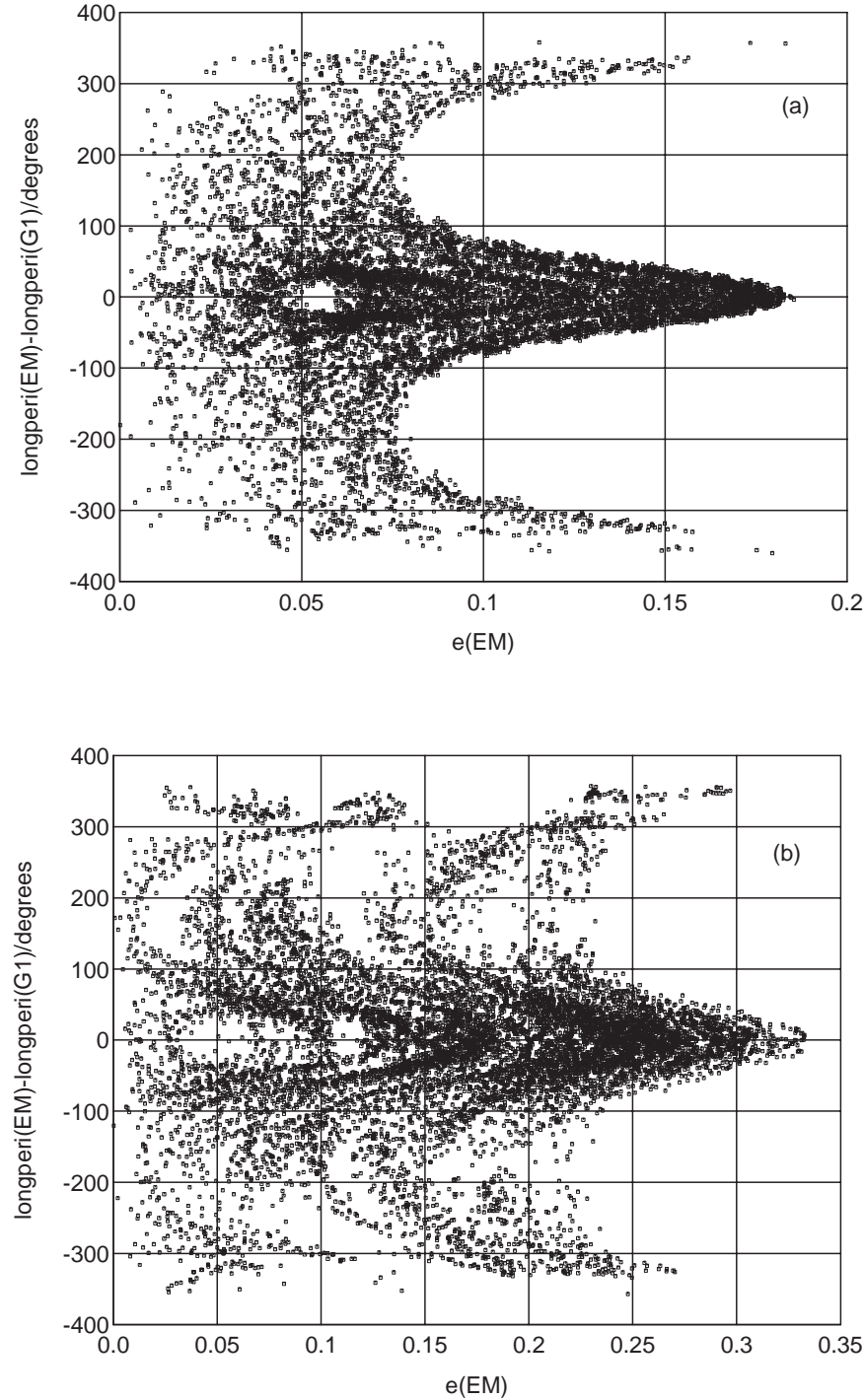

Fig. 5. Different degrees of coupling between $e_{\mathrm{EM}}$ and $\Delta \varpi_{\mathrm{EM} 1}$ in the 47 UMa system a) relatively strong coupling, associated with relative stability (row 25 in Table 3a) b) weaker coupling, associated with relative instability (row 28 in Table $3 a$ ).

maxima. The atmosphere would have to be sufficiently massive to screen the surface from temperature variations that would be inimical to life. We know of no detailed attempts to model the required atmosphere.

\subsection{The fate of EM at minimum giant masses}

From our results at 1.5 times the minimum masses we know that $\left|\Delta \varpi_{21}(0)\right|=120^{\circ}$ is usually the least stable configuration (and that $i_{\mathrm{EM}}(0)$ up to at least $10^{\circ}$ and the value of $\varpi_{\mathrm{EM}}(0)$ make little difference). Therefore, at minimum mass we usually ran configurations other than $\left|\Delta \varpi_{21}(0)\right|=120^{\circ}$ only if this configuration was unstable - if it was stable then the other configurations are likely to be stable too.

Table 4 and the lower half of Fig. 6 summarise our results at the minimum giant masses. As expected, we now find stable orbits over more of the habitable zones than at 1.5 times the minimum giant masses. There is a slight curiosity at $0.85 \mathrm{AU}$,

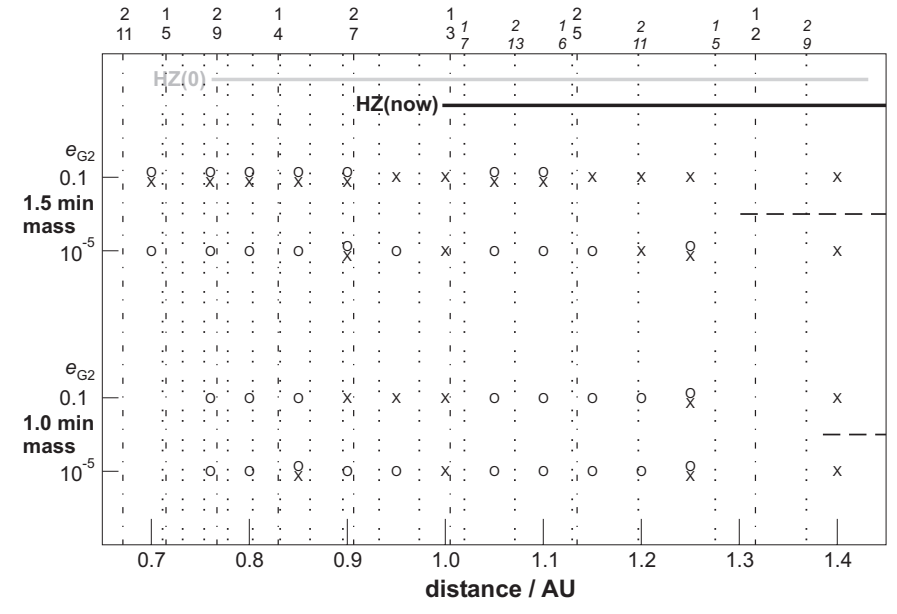

Fig. 6. Stable and unstable orbits of an Earth-mass planet (EM) in the 47 UMa system. The distance is that of EM from the star. The crosses denote orbits of EM that lead to close encounters with the inner giant (G1) within $1000 \mathrm{Ma}$. The open circles denote orbits that avoid close encounters for the full $1000 \mathrm{Ma}$ of the integration. Further details are in Sects. 4.2 and 4.3.

namely that $e_{\mathrm{G} 2}(0)=0.1$ runs for $>1000 \mathrm{Ma}$, but $e_{\mathrm{G} 2}(0)=10^{-5}$ has mixed fortunes. It is perhaps not surprising that a system with two giants and EM is sufficiently complex for there to be islands of instability in the multi-dimensional space of the launch configuration. The immediate cause is weaker $e_{\mathrm{EM}}-$ $\Delta \varpi_{\mathrm{EM} 1}$ coupling in the $e_{\mathrm{G} 2}(0)=10^{-5}$ case.

\subsection{The fate of EM beyond $1000 \mathrm{Ma}$}

At minimum masses and at 1.5 times these values, there are only a few cases where the orbit becomes unstable after $500 \mathrm{Ma}$. Moreover, $e_{\mathrm{EM}}(t)$ in such cases displays large variations on a $50 \mathrm{Ma}$ timescale with a tendency for the maximum values of $e_{\mathrm{EM}}(t)$ to increase, as exemplified in Fig. 7a (which corresponds to row 8 in Table 3a). By contrast the great majority of runs that last for the full 1000 Ma display no such trends, as illustrated in Fig. $7 \mathrm{~b}$ (which corresponds to row 12 in Table 3a). Orbits that last for at least $1000 \mathrm{Ma}$ ("stable" orbits) are thus likely to last for very much longer.

\subsection{What chance an Earth mass planet in $\mathrm{HZ}$ (now)?}

At both sets of giant masses, orbits of EM that last for at least $1000 \mathrm{Ma}$ are found in all but the outer part of $\mathrm{HZ}(0)$ and in the inner part of $\mathrm{HZ}$ (now), particularly if the eccentricity of G2 is much less than 0.1 with that of G1 at its present value of 0.061 . If this condition is not met then stable orbits can be found only for certain combinations of the longitudes of periastron at $t=0$. We have begun to investigate the reasons for this, and further studies are in progress. Here our main goal has been to determine the sort of parameter ranges at which stable orbits might be found, and hence to establish whether there could be Earthmass planets in HZ(now) - it seems there could be.

We can use Fig. 6 as the basis of a rough estimate of the chance of finding an Earth-mass planet in HZ(now) of 47UMa, assuming that such a planet could have formed. We accept only 
Table 4. Orbital integrations for 47 UMa with two giants present, minimum mass.

\begin{tabular}{|c|c|c|c|c|c|c|c|c|c|}
\hline \multicolumn{7}{|c|}{ input at $t=0$} & \multicolumn{3}{|c|}{ outcome } \\
\hline row & $a_{\mathrm{EM}}$ & $i_{\mathrm{EM}}$ & $e_{\mathrm{G} 2}$ & $\varpi_{\mathrm{EM}}$ & $\varpi_{\mathrm{G} 1}$ & $\varpi_{\mathrm{G} 2}$ & enc'nter & range & range \\
\hline no. & /AU & $1^{\circ}$ & $1^{\circ}$ & $1^{\circ}$ & $1^{\circ}$ & $1^{\circ}$ & $/ \mathrm{Ma}$ & $a_{\mathrm{EM}} / \mathrm{AU}$ & $e_{\mathrm{EM}}$ \\
\hline 1 & 1.40 & 0 & $10^{-5}$ & 0 & 180 & 90 & 9.0 years & $1.400-1.414$ & $10^{-5}-0.048$ \\
\hline 2 & 1.40 & $\mathbf{0}$ & 0.1 & 0 & 120 & 240 & 8.4 years & $1.400-1.412$ & $10^{-5}-0.0438$ \\
\hline 3 & 1.25 & 0 & $10^{-5}$ & 0 & 180 & 90 & $>1000$ & $1.244-1.256$ & $10^{-5}-0.048$ \\
\hline 4 & 1.25 & 0 & $10^{-5}$ & 0 & 120 & 240 & 48.1 & $1.240-1.269$ & $10^{-5}-0.199$ \\
\hline 5 & 1.25 & 0 & 0.1 & 0 & 180 & 90 & $>1000$ & $1.243-1.257$ & $10^{-5}-0.106$ \\
\hline 6 & 1.25 & 0 & 0.1 & 0 & 120 & 240 & 4.72 & $1.240-1.264$ & $10^{-5}-0.197$ \\
\hline 7 & 1.20 & 0 & $10^{-5}$ & 0 & 120 & 240 & $>1000$ & $1.198-1.206$ & $10^{-5}-0.095$ \\
\hline 8 & 1.20 & 0 & 0.1 & 0 & 120 & 240 & $>1000$ & $1.198-1.206$ & $10^{-5}-0.119$ \\
\hline 9 & 1.15 & 0 & $10^{-5}$ & 0 & 120 & 240 & $>1000$ & $1.148-1.155$ & $10^{-5}-0.102$ \\
\hline 10 & 1.15 & 0 & 0.1 & 0 & 120 & 240 & $>1000$ & $1.148-1.155$ & $10^{-5}-0.136$ \\
\hline 11 & 1.10 & 0 & $10^{-5}$ & 0 & 120 & 240 & $>1000$ & 1.099-1.104 & $10^{-5}-0.104$ \\
\hline 12 & 1.10 & 0 & 0.1 & 0 & 120 & 240 & $>1000$ & $1.098-1.104$ & $10^{-5}-0.166$ \\
\hline 13 & 1.05 & 0 & $10^{-5}$ & 0 & 120 & 240 & $>1000$ & $1.049-1.053$ & $10^{-5}-0.111$ \\
\hline 14 & 1.05 & 0 & 0.1 & 0 & 120 & 240 & $>1000$ & $1.049-1.058$ & $10^{-5}-0.201$ \\
\hline 15 & 1.00 & 0 & $10^{-5}$ & 0 & 180 & 90 & 551 & $0.987-1.020$ & $10^{-5}-0.485$ \\
\hline 16 & 1.00 & 0 & 0.1 & 0 & 180 & 90 & 6.96 & $0.985-1.069$ & $10^{-5}-0.670$ \\
\hline 17 & 0.95 & 0 & $10^{-5}$ & 0 & 120 & 240 & $>1000$ & $0.949-0.952$ & $10^{-5}-0.123$ \\
\hline 18 & 0.95 & 0 & 0.1 & 0 & 180 & 90 & 0.233 & $0.949-1.066$ & $10^{-5}-0.658$ \\
\hline 19 & 0.95 & 0 & 0.1 & 0 & 120 & 240 & $0.013(\mathrm{G} 2)$ & $0.950-2.661$ & $10^{-5}-0.914$ \\
\hline 20 & 0.90 & 0 & $10^{-5}$ & 0 & 120 & 240 & $>1000$ & 0.899-0.902 & $10^{-5}-0.209$ \\
\hline 21 & 0.90 & 0 & 0.1 & 0 & 180 & 90 & 0.024 & $0.900-0.961$ & $10^{-5}-0.631$ \\
\hline 22 & 0.90 & 0 & 0.1 & 0 & 120 & 240 & 874 & $0.896-0.973$ & $10^{-5}-0.600$ \\
\hline 23 & 0.85 & 0 & $10^{-5}$ & 0 & 180 & 90 & $>1000$ & $0.846-0.853$ & $10^{-5}-0.402$ \\
\hline 24 & 0.85 & 0 & $10^{-5}$ & 0 & 120 & 240 & 5.45 & $0.839-0.955$ & $10^{-5}-0.628$ \\
\hline 25 & 0.85 & 0 & 0.1 & 0 & 120 & 240 & $>1000$ & $0.850-0.851$ & $10^{-5}-0.130$ \\
\hline 26 & 0.80 & 0 & 0 & 0 & 120 & 240 & $>1000$ & $0.800-0.801$ & $10^{-5}-0.0860$ \\
\hline 27 & 0.80 & 0 & 0.1 & 0 & 120 & 240 & $>1000$ & $0.800-0.801$ & $10^{-5}-0.0899$ \\
\hline 28 & 0.76 & 0 & $10^{-5}$ & 0 & 120 & 240 & $>1000$ & $0.760-0.761$ & $10^{-5}-0.0548$ \\
\hline 29 & 0.76 & 0 & 0.1 & 0 & 120 & 240 & $>1000$ & $0.760-0.761$ & $10^{-5}-0.0773$ \\
\hline
\end{tabular}

those values of $a_{\mathrm{EM}}(0)$ at which there is stability at all angular configurations investigated. We exclude ranges of $a_{\mathrm{EM}}(0)$ centred on mean motion resonances - our earlier work (Jones et al. 2001) shows that mean motion resonances in the inner region of $\mathrm{HZ}$ (now) of 47UMa are about $0.02 \mathrm{AU}$ wide. $\mathrm{HZ}$ (now) extends from 1.00-1.90 AU (Fig. 1), an interval of $0.90 \mathrm{AU}$. The best case is with the giants at their minimum mass and with $e_{\mathrm{G} 2}(0)=10^{-5}$. The possibly stable sub-range is approximately an interval of $0.20 \mathrm{AU}$, but we have to exclude five mean motion strips $0.02 \mathrm{AU}$ wide, leaving intervals summing to $0.10 \mathrm{AU}$. Thus, the proportion of HZ(now) that could have an Earth-mass planet is roughly $0.10 / 0.90$, which is $11 \%$. Proceeding in this way for the other three cases in Fig. 6 we obtain

- giants at their minimum mass $e_{\mathrm{G} 2}(0)=0.1,11 \%$;

- giants at 1.5 times minimum mass, $e_{\mathrm{G} 2}(0)=10^{-5}, 8 \%$;

- giants at 1.5 times minimum mass, $e_{\mathrm{G} 2}(0)=0.1,0 \%$.

\section{Comparison with other work}

Fischer et al. (2002) have also investigated the upper limit on $e_{\mathrm{G} 2}$ in the absence of any Earth-mass bodies. They integrated 

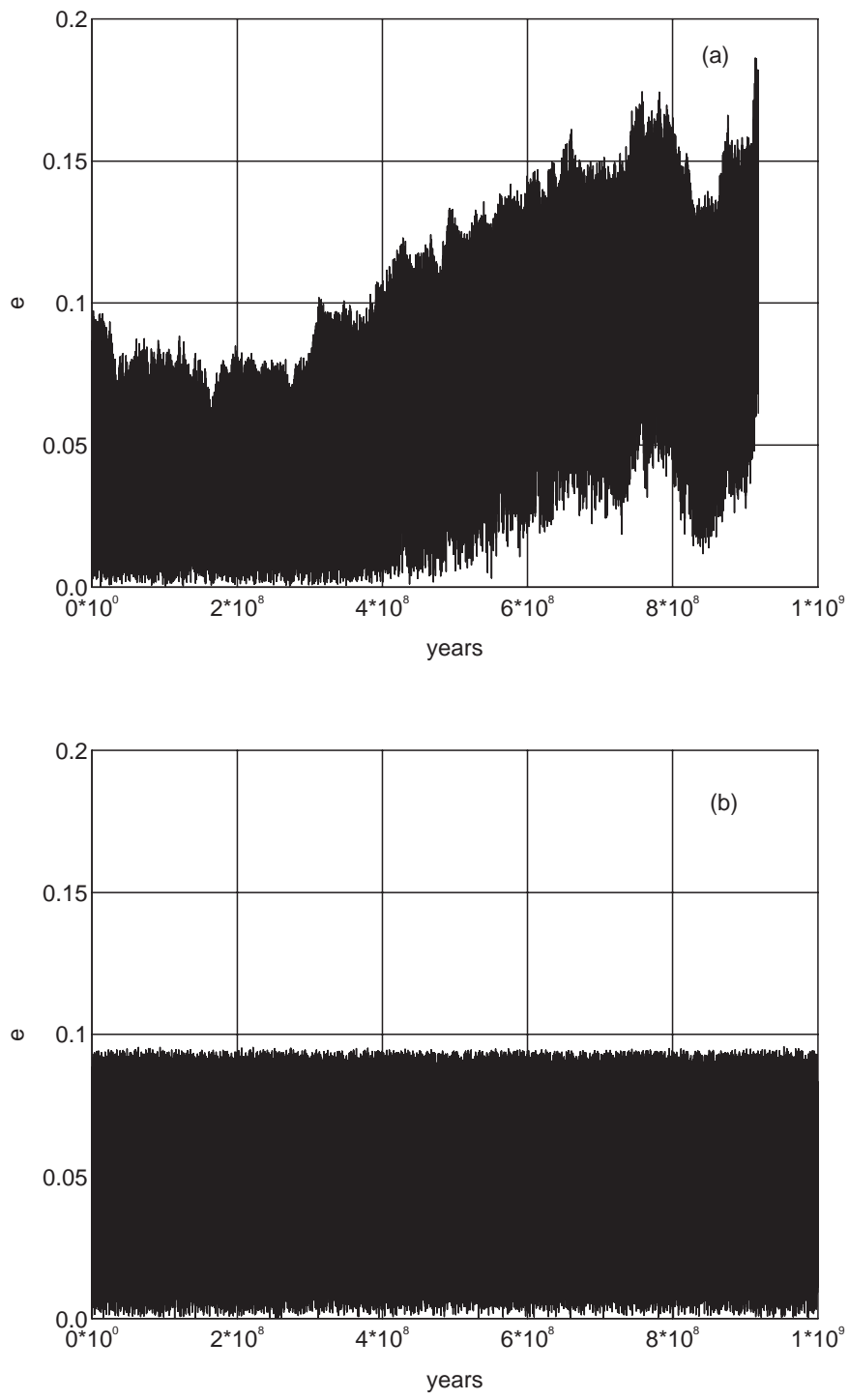

Fig. 7. The behaviour of the eccentricity of an Earth-mass planet in stable and unstable cases. a) An unstable case, in that there is a close encounter within $1000 \mathrm{Ma}$, at an unusually late time. It corresponds to row 8 in Table 3a. b) A stable case, in that there are no close encounters within the $1000 \mathrm{Ma}$ of the integration. This is typical of integrations that last the full $1000 \mathrm{Ma}$. It corresponds to row 12 in Table 3 a.

for up to $100 \mathrm{Ma}$ with a Bulirsch-Stoer integrator and were thus able to follow the encounters beyond the $3 R_{\mathrm{H}}$ point at which we stopped integrating. Close encounters resulted in ejection of G2. For our work Fig. 3 shows a typical end-point of the close encounter in row 11 in Table 2. You can see that the sharp increase in $e_{\mathrm{G} 2}(t)$ at the encounter makes it likely that ejection would have followed, so our criterion is not particularly conservative in this respect. Fischer et al. report the largest value of $e_{\mathrm{G} 2}$ achieved during each integration that lasted for $100 \mathrm{Ma}$. They neglected correlations between the eccentricities and so their values are to be compared with the largest of our three values at each mass. At 1.5 times the minimum giant masses they obtain 0.16 , much the same as our value of 0.163 . Laughlin et al. (2002) by a similar procedure obtained 0.154 .
At minimum masses Fischer et al. (2002) get 0.22, Laughlin et al. (2002) get 0.198 , and we get 0.172 . We presume that the difference lies partly in our more conservative definition of a close encounter, partly in their sole use of the currently observed difference between $\varpi_{\mathrm{G} 1}$ and $\varpi_{\mathrm{G} 2}$ of $45^{\circ}$, which avoids the instability at a difference of $120^{\circ}$, and partly in their shorter integration time - $100 \mathrm{Ma}$ versus $1000 \mathrm{Ma}$ in our work, which misses the cases of instability that arise after $100 \mathrm{Ma}$.

Turning to EM, in our earlier paper (Jones et al. 2001) we reported stable confined orbits for launches of EM across more of the inner regions of $\mathrm{HZ}(0)$ and $\mathrm{HZ}$ (now) than we find in this work. In our earlier work the outer giant was unknown and so we only had the inner giant. To show that this makes the difference we re-ran a few of the unstable orbits in Tables 3a and $b$ with no G2, and the integration then ran for $1000 \mathrm{Ma}$. $\mathrm{G} 2$ causes significant excursions in $e_{\mathrm{G} 1}(t)$ (Table 2), and this surely contributes to this loss of stability in EM.

Laughlin et al. (2002) have looked briefly at the fate of mass-less particles launched in the $47 \mathrm{UMa}$ system with the two giants at minimum mass. They launched 280 particles in low-inclination, low-eccentricity orbits with semimajor axes in the range $0.4 \mathrm{AU}$ to $2.0 \mathrm{AU}$. The integrations were for only $20 \mathrm{Ma}$, and they used a hybrid integrator that could handle close encounters. The outcome is in broad agreement with the lower part of Fig. 6, though the fate of massless particles over $20 \mathrm{Ma}$ is not obviously indicative of the fate of Earth-mass bodies over $1000 \mathrm{Ma}$.

Noble et al. (2002) have used numerical integration to study the orbital stability of a terrestrial body in the 47 UMa system with 1.44 times the minimum giant masses and $e_{\mathrm{G} 2}=0.005$. They launched the "Earth" at $a_{\mathrm{E}}(0)=1.128 \mathrm{AU}, 1.44 \mathrm{AU}$, and $1.752 \mathrm{AU}$, with $\varpi(0)=0$ for all three planets. Their integration times are short, hundreds of thousands of years. They find stability over such durations only at $a_{\mathrm{E}}(0)=1.28 \mathrm{AU}$. Line 12 in Table $3 \mathrm{a}$ is our most similar case, and this too is stable, in fact for $>1000 \mathrm{Ma}$. Based on our results at $a_{\mathrm{EM}}(0)>1.25 \mathrm{AU}$, we expect lifetimes at $a_{\mathrm{E}}(0)=1.44 \mathrm{AU}$, and $1.752 \mathrm{AU}$ to be much less than $1 \mathrm{Ma}$, in agreement with their work.

\section{Conclusions}

1. At the present epoch, when the inner giant has an orbital eccentricity of $0.061 \pm 0.014$, the eccentricity of the outer giant is unlikely to be much greater than about 0.15 at minimum giant mass, and about 0.13 at masses of 1.5 times this minimum.

2. Whereas the $47 \mathrm{UMa}$ system as previously known, with just one giant planet, could have had Earth-mass planets confined to the habitable zone for a fairly wide range of initial orbital parameters, the second (outer) giant now known to be present has reduced the range significantly.

3. Provided that Earth-mass planets can have formed interior to the inner giant in the $47 \mathrm{UMa}$ system (and this seems to be possible), it is likely that they will still be present today under the following circumstances (Fig. 6). First, they must be launched within about 1.2 AU of the star, which 
includes the inner part of the present-day habitable zone, second, they must be away from mean-motion resonances, Third, one or more of the following conditions must be met.

(i) The present eccentricity of the outer giant is considerably less than 0.1 .

(ii) The giant masses are much closer to their minimum values than to 1.5 times these values.

Under these conditions the order of $10 \%$ of the present habitable zone could contain stable, confined orbits. If neither of conditions (i) and (ii) is met then stable orbits for Earth-mass planets can be found only for certain combinations of the longitudes of perihelion at $t=0$. Though the immediate causes include varying degrees of $e_{\mathrm{EM}}-\Delta \varpi_{\mathrm{EM} 1}$ coupling, such causes were not the primary goal of this study, but are the subject of further, possibly lengthy, investigation.

4. When it becomes possible to detect terrestrial planets in exoplanetary systems, the $47 \mathrm{UMa}$ system will be well worth attention. Earth-mass planets could be present close to the star and in the inner region of the habitable zone.

Acknowledgements. We are grateful to Professor Mark Bailey and Dr John Chambers for discussions, and to an anonymous referee for useful comments.

\section{References}

Boss, A. P. 1995, Science, 267, 360

Chambers, J. E. 1999, MNRAS, 304, 793

Charbonneau, D., Brown, T. M., Latham, D. W., \& Mayor, M. 2000, ApJ, 529, L45

Fischer, D. A., Marcy, G. W., Butler, R. P., Laughlin, G., \& Vogt, S. S. 2002, ApJ, 5641028

Forget, F., \& Pierrehumbert, R. T. 1997, Science, 278, 1273

Gonzalez, G. 1998, A\&A, 334, 221

Jones, B. W., Sleep, P. N., \& Chambers, J. E. 2001, A\&A, 366, 254

Kasting, J. F., Whitmire, D. P., \& Reynolds, R. T. 1993, Icarus, 101, 108

Laughlin, G., Chambers, J. E., \& Fischer, D. A. 2002, accepted for ApJ

Levison, H. F., \& Duncan, M. J. 1994, Icarus, 108, 18

Lin, D. N. C., Papaloizou, J. C. B, Terquem, C., Bryden, G., \& Ida, S. 2000, Protostars and Planets, IV, 1111

Lissauer, J. J. 1987, Icarus, 69, 249

Masset, F., \& Snellgrove, M. 2001, MNRAS, 320, L55

Murray, N., Hansen, B., Holman, M., \& Tremaine, S. 1998, Science, 279, 69

Noble, M., Musielak, Z. E., \& Cuntz, M. 2002, ApJ, 572, 1024

Perryman, M. A. C., Lindegren, L., Arenou, F., et al. 1996, A\&A, 310, L21

Pollack, J. B., Hubickyj, O., Bodenheimer, P., et al. 1996, Icarus, 124, 62

Pourbaix, D. 2001, A\&A, 323, L22

Schneider, J., 2002, http://www. obspm. fr/encycl/catalog.html Ward, W. R., \& Hahn, J. M. 2000, Protostars and Planets, IV, 1135 